\title{
Transdermal Delivery of Small Interfering RNA with Elastic Cationic Liposomes in Mice
}

\author{
Yoshiyuki Hattori, Masataka Date, Shohei Arai, Kumi Kawano, \\ Etsuo Yonemochi, and Yoshie Maitani \\ Institute of Medicinal Chemistry, Hoshi University, Ebara 2-4-41, Shinagawa-ku, Tokyo 142-8501, Japan \\ Correspondence should be addressed to Yoshiyuki Hattori; yhattori@hoshi.ac.jp
}

Received 4 October 2013; Revised 27 November 2013; Accepted 3 December 2013

Academic Editor: Hidetaka Akita

Copyright (C) 2013 Yoshiyuki Hattori et al. This is an open access article distributed under the Creative Commons Attribution License, which permits unrestricted use, distribution, and reproduction in any medium, provided the original work is properly cited.

\begin{abstract}
We developed elastic cationic liposomal vectors for transdermal siRNA delivery. These liposomes were prepared with 1,2-dioleoyl3-trimethylammonium-propane (DOTAP) as a cationic lipid and sodium cholate (NaChol) or Tween 80 as an edge activator. When NaChol or Tween 80 was included at 5, 10, and 15\% (w/w) into DOTAP liposomal formulations (C5-, C10-, and C15liposomes and T5-, T10-, and T15-liposomes), C15- and T10-liposomes showed 2.4- and 2.7-fold-higher elasticities than DOTAP liposome, respectively. Although the sizes of all elastic liposomes prepared in this study were about $80-90 \mathrm{~nm}$, the sizes of C5-, C10- and C15-liposome/siRNA complexes (lipoplexes) were about 1,700-1,800 nm, and those of T5-, T10-, and T15-lipoplexes were about $550-780 \mathrm{~nm}$. Their elastic lipoplexes showed strong gene suppression by siRNA without cytotoxicity when transfected into human cervical carcinoma SiHa cells. Following skin application of the fluorescence-labeled lipoplexes in mice, among the elastic lipoplexes, C15- and T5-lipoplexes showed effective penetration of siRNA into skin, compared with DOTAP lipoplex and free siRNA solution. These data suggest that elastic cationic liposomes containing an appropriate amount of NaChol or Tween 80 as an edge activator could deliver siRNA transdermally.
\end{abstract}

\section{Introduction}

RNA interference (RNAi) offers potential for treating a wide variety of disorders through selective silencing of diseaserelevant RNAs $[1,2]$. Topical use of small interfering RNA (siRNA) has been increasingly studied for its applicability in treating skin disease [3]. However, transdermal naked siRNA delivery is limited due to its low permeability of skin barriers such as the stratum corneum and the epidermal layer.

Liposomes have been widely investigated in dermatology as transdermal carrier systems [4]. Of particular interest are cationic liposomes for passive transdermal delivery of siRNA, since their opposite charges spontaneously result in complexation due to electrostatic interactions. Cationic liposomes protect siRNA from degradation and allow the introduction of siRNA into cells. It has been reported that elastic cationic liposomes (ultradeformable cationic liposomes) can penetrate intact skin while carrying plasmid DNA (pDNA) [5] and antisense oligonucleotides [6] when applied under nonoccluded conditions. Elastic cationic liposomes consist of a cationic lipid and an edge activator that is responsible for the elasticity of the membrane. An edge activator is usually a single-chain surfactant that destabilizes the lipid bilayers of the liposomes and increases the deformability of the bilayers [7]. For transdermal gene delivery, sodium cholate ( $\mathrm{NaChol}$ ), sodium deoxycholate ( $\mathrm{NaDChol})$, and Tween 80 were used as an edge activator $[5,6,8]$; however, there is little information about the feasibility and effect of an edge activator on elastic liposomes for transdermal siRNA delivery in vivo.

Deformability of elastic liposome enables therapeutic siRNA to penetrate the skin barrier by the stratum corneum. A cationic lipid, 1,2-dioleoyl-3-trimethylammonium propane (DOTAP), has frequently been used as a cationic lipid for a liposomal delivery system of siRNA by several research groups [9-12]. Moreover, we previously reported that $\mathrm{NaChol}$ and Tween 80 incorporated into liposomal membranes confer deformation and flexibility to ultradeformable liposomes $[13,14]$. Therefore, in the present study, for transdermal 
TABLE 1: Particle size and $\zeta$-potential of liposomes and lipoplexes used in this study.

\begin{tabular}{|c|c|c|c|c|c|c|c|c|}
\hline \multirow{2}{*}{ Vesicles } & \multicolumn{3}{|c|}{$\%(w / w)$} & \multicolumn{3}{|c|}{ Liposome $^{a}$} & \multicolumn{2}{|c|}{ Lipoplex $^{\mathrm{a}, \mathrm{b}}$} \\
\hline & DOTAP & $\mathrm{NaChol}^{\mathrm{c}}$ & Tween 80 & Size (nm) & $\mathrm{PD}^{\mathrm{d}}$ & $\zeta$-potential $(\mathrm{mV})$ & Size $(\mathrm{nm})$ & $\mathrm{PD}^{\mathrm{d}}$ \\
\hline DOTAP & 100 & - & - & $109 \pm 2$ & $0.10 \pm 0.02$ & $40.2 \pm 2.4$ & $1724 \pm 123$ & $0.63 \pm 0.04$ \\
\hline $\mathrm{C} 5$ & 95 & 5 & - & $84 \pm 2$ & $0.11 \pm 0.02$ & $42.4 \pm 1.4$ & $1795 \pm 432$ & $0.65 \pm 0.13$ \\
\hline $\mathrm{C} 10$ & 90 & 10 & - & $87 \pm 3$ & $0.16 \pm 0.00$ & $52.2 \pm 2.2$ & $1809 \pm 161$ & $0.61 \pm 0.05$ \\
\hline $\mathrm{C} 15$ & 85 & 15 & - & $82 \pm 1$ & $0.13 \pm 0.01$ & $47.8 \pm 1.7$ & $1859 \pm 115$ & $0.56 \pm 0.02$ \\
\hline T5 & 95 & - & 5 & $82 \pm 1$ & $0.19 \pm 0.01$ & $36.0 \pm 0.8$ & $582 \pm 138$ & $0.26 \pm 0.04$ \\
\hline T10 & 90 & - & 10 & $87 \pm 1$ & $0.17 \pm 0.01$ & $26.4 \pm 1.8$ & $550 \pm 45$ & $0.26 \pm 0.01$ \\
\hline $\mathrm{T} 15$ & 85 & - & 15 & $83 \pm 2$ & $0.15 \pm 0.01$ & $30.8 \pm 1.5$ & $775 \pm 106$ & $0.33 \pm 0.04$ \\
\hline
\end{tabular}

${ }^{\mathrm{a}}$ In water. ${ }^{\mathrm{b}}$ Weight ratio of lipid/siRNA $=14 / 1 .{ }^{\mathrm{c}} \mathrm{NaChol}$, sodium cholate. ${ }^{\mathrm{d}} \mathrm{PD}$, polydispersity index. Values represent means \pm S.D. $(n=3)$.

siRNA delivery, we optimized the formulation of elastic DOTAP liposomes including $\mathrm{NaChol}$ or Tween 80 , which are positively charged liposomes exhibiting some deformability, and evaluated the permeability of siRNA in mouse skin after topical application of elastic liposome/siRNA complex (elastic lipoplex).

\section{Materials and Methods}

2.1. Materials. 1,2-Dioleoyl-3-trimethylammonium propane methyl sulfate (DOTAP) was purchased from Avanti Polar Lipids Inc. (Albaster, AL, USA). Sodium cholate hydrate (NaChol) was purchased from Sigma-Aldrich Japan (Tokyo, Japan). Tween 80 was purchased from NOF Inc. (Tokyo, Japan). Lissamine rhodamine B 1,2-dihexadecanoyl-snglycero-3-phosphoethanolamine, triethylammonium salt (rhodamine-DHPE), was purchased from Invitrogen (Carlsbad, CA, USA). All other chemicals were of the finest grade available.

2.2. siRNA. The siRNAs targeting nucleotides of firefly luciferase (Luc siRNA) and nonsilencing siRNA (Cont siRNA) as a negative control were synthesized by Sigma Genosys (Tokyo, Japan) [15]. The siRNA sequences of the Luc siRNA were passenger strand: $5^{\prime}$-CCGUGGUGUUCGUGUCUAAGA-3' and guide strand: $5^{\prime}$-UUAGACACGAACACCACGGUA-3. The siRNA sequences of the Cont siRNA were passenger strand: $5^{\prime}$-CCGUACUAGCCAUUAUGCGUC- $3^{\prime}$ and guide strand: $5^{\prime}$-CGCAUAAUGGCUAGUACGGGU-3' . 6-Carboxyfluorescein-aminohexylamidite- (FAM-) labeled luciferase (pGL3) siRNA was obtained from Cosmo Bio Co., Ltd. (Tokyo, Japan). The siRNA sequences of the FAM-labeled luciferase siRNA were passenger strand: $5^{\prime}$-UCGAAGUACUCAGCGUAAGdTdT$3^{\prime}$ and guide strand: $5^{\prime}$-CUUACGCUGAGUACUUCGAdT$\mathrm{dT}-3^{\prime}$.

2.3. Preparation of Liposomes and Lipoplexes. NaChol and Tween 80 were dissolved in phosphate-buffered saline (PBS, $\mathrm{pH}$ 7.4) by vortexing. DOTAP liposomes were prepared by vortexing in PBS with a concentration of $10 \mathrm{mg} / \mathrm{mL}$ DOTAP until a milky suspension was obtained as previously reported [6]. Elastic liposomes were prepared by vortexing after adding $\mathrm{NaChol}$ or Tween 80 solution to DOTAP solution at final concentrations of 5, 10, and 15\% (w/w) (Table 1). For preparation of rhodamine-labeled liposomes, rhodamineDHPE was incorporated into the liposomal formulation at $0.2 \mathrm{~mol} \%$ in the total lipid. The liposomes were sonicated in a bath-type sonicator (Branson 25010J-MTH, USA) for $1 \mathrm{~h}$ at room temperature and then filtered 31 times through $100 \mathrm{~nm}$ polycarbonate membrane filters (Whatman, Brentfort, UK).

Lipoplexes were prepared by mixing the liposome with siRNA at a weight ratio of DOTAP/siRNA of 14/1 (charge ratio $(+/-)$ of $6 / 1)$, as previously reported [9], and then incubated at room temperature for $10-15 \mathrm{~min}$. Average diameters and $\zeta$-potentials of liposomes and lipoplexes were measured by dynamic light-scattering and electrophoresis light-scattering methods, respectively (ELS-Z2; Otsuka Electronics, Osaka, Japan). All measurements were performed at $25 \pm 1^{\circ} \mathrm{C}$, after diluting the liposome and lipoplex suspension with Milli-Q water.

2.4. Measurement of Elasticity. The elasticity of the bilayer of liposomes was directly proportional to $J_{\text {flux }} \times\left(r_{v} / r_{p}\right)^{2}$ :

$$
\text { Elasticity }\left(\mu \mathrm{g} \cdot \mathrm{sec}^{-1} \cdot \mathrm{cm}^{-2}\right)=J_{\text {flux }} \times\left(\frac{r_{v}}{r_{p}}\right)^{2},
$$

where $J_{\text {flux }}$ is the rate of penetration through a permeability barrier, $r_{v}$ is the size of liposomes after extrusion, and $r_{p}$ is the pore size of the barrier:

$$
\begin{aligned}
J_{\text {flux }} & \left(\mu \mathrm{g} \cdot \mathrm{sec}^{-1} \cdot \mathrm{cm}^{-2}\right) \\
= & J \frac{(\operatorname{DOTAP}(\mu \mathrm{g}))}{\left(\text { extrusion time }(\mathrm{sec}) \times \text { membrane area }\left(\mathrm{cm}^{2}\right)\right) .}
\end{aligned}
$$

To measure $J$, the liposomes were extruded through a polycarbonate membrane $\left(4.9 \mathrm{~cm}^{2}\right)$ with a pore diameter of $50 \mathrm{~nm}\left(r_{p}\right)$, at a pressure of $0.5 \mathrm{MPa}$. After $10 \mathrm{~min}$ of extrusion, the extrudate was weighed, and then concentration of DOTAP in the extrudate was calculated by measurement of absorbance at $228 \mathrm{~nm}$ after the addition of equal volume of methanol. The average liposome diameter after extrusion $\left(r_{v}\right)$ was measured by ELS-Z2 as described in the above section.

2.5. Cell Culture. Human cervical carcinoma SiHa cells stably expressing firefly luciferase (FL-SiHa) were donated by 
Dr. Kenji Yamato (Department of Gastroenterology, Tsukuba University, Tsukuba, Japan). FL-SiHa cells were grown in Eagle's MEM, supplemented with 10\% heat-inactivated fetal bovine serum (FBS), $100 \mu \mathrm{g} / \mathrm{mL}$ kanamycin, and $1 \mathrm{mg} / \mathrm{mL}$ $\mathrm{G} 418$ at $37^{\circ} \mathrm{C}$ in a $5 \% \mathrm{CO}_{2}$ humidified atmosphere.

2.6. Luciferase Activity. FL-SiHa cells were seeded at a density of $4 \times 10^{5}$ cells per well in 24 -well plates and maintained for $24 \mathrm{~h}$ before transfection. For transfection, each lipoplex of siRNA was diluted with serum-free medium to a final concentration of $50 \mathrm{nM}$ Luc or Cont siRNA and then gently added to the cells. After incubation for $3 \mathrm{~h}$ at $37^{\circ} \mathrm{C}$, the cells were added with FBS to a final concentration of $10 \%$ and then incubated for another $45 \mathrm{~h}$. Lipofectamine RNAiMax lipoplex (Invitrogen Corp.) was prepared according to the manufacturer's protocol. Forty-eight hours after the transfection, luciferase activity was measured as counts per sec (cps)/ $\mu$ g protein using the luciferase assay system (Promega, Madison, USA) and BCA reagent (Pierce, Rockford, IL, USA), as previously reported [15].

2.7. Cytotoxicity. FL-SiHa cells were seeded at a density of $6 \times$ $10^{4}$ cells per well in 96-well plates and maintained for $24 \mathrm{~h}$ before transfection. For transfection, each lipoplex of siRNA was diluted with serum-free medium to a final concentration of $50 \mathrm{nM}$ Cont siRNA and then gently added to the cells. After incubation for $3 \mathrm{~h}$ at $37^{\circ} \mathrm{C}$, the cells were supplemented with FBS to a final concentration of $10 \%$. After $45 \mathrm{~h}$ of incubation, the medium was removed, and the cells were treated with WST-8 (2-(2-methoxy-4-nitrophenyl)-3-(4-nitrophenyl)-5(2,4-disulfophenyl)-2H-tetrazolium, monosodium salt) solution $(10 \mu \mathrm{L})$ in medium containing serum $(100 \mu \mathrm{L})$ for $30 \mathrm{~min}$. Cell viability is expressed relative to the absorbance at $450 \mathrm{~nm}$ of untransfected cells.

2.8. Skin Penetration of Liposomes and Lipoplexes. The excised skin of hairless mice (Laboskin, HOS: HR-1 male, 7 weeks, Hosino Laboratory Animals, Inc., Ibaraki, Japan) was used for the investigation of skin permeability by elastic liposomes. For this purpose, rhodamine-labeled liposomes were nonocclusively applied on the Laboskin $\left(3.14 \mathrm{~cm}^{2}\right)$ for $6 \mathrm{~h}$.

Female HR-1/Hos hairless mice (6 weeks of age) were purchased from Hosino Laboratory Animals, Inc. All in vivo experiments were approved by the Institutional Animal Care and Use Committee of Hoshi University. For observation of the skin penetration of the elastic lipoplexes, the lipoplexes of rhodamine-labeled liposomes and FAM-labeled luciferase siRNA $(50 \mu \mathrm{g})$ were applied nonocclusively on the dorsal skin $\left(3.14 \mathrm{~cm}^{2}\right)$ under anesthesia for $6 \mathrm{~h}$. The mice were kept on a hot plate during the sedation period.

After application with elastic liposomes or lipoplexes, the skins were embedded in OCT compound (Tissue-Tek, Sakura Finetechnical Co., Ltd., Tokyo, Japan) and processed by frozen sectioning at $20 \mu \mathrm{m}$. Each frozen section was mounted on a silane-coating slide (Muto Pure Chemicals Co., Ltd., Tokyo, Japan). Localizations of FAM-labeled siRNA and rhodamine-labeled liposomes were examined using an LSM5 EXCITER confocal laser scanning microscope (Carl
Zeiss, Thornwood, NY, USA). FAM-labeled siRNA was imaged using an argon laser at $488 \mathrm{~nm}$ excitation, and fluorescence emission was observed with a filter, BP505530. For rhodamine-labeled liposome, maximal excitation was performed with a $543 \mathrm{~nm}$ internal He-Ne laser, and fluorescence emission was observed with an LP560.

2.9. Statistical Analysis. The statistical significance of differences between mean values was determined by using Student's $t$-test. A $P$ value of 0.05 or less was considered significant.

\section{Results and Discussion}

Transdermal delivery of naked siRNA is limited due to its low stability in skin and low permeability by various skin barriers such as the stratum corneum and epidermal layer. In this study, we investigated whether cationic elastic liposomes could effectively deliver siRNA transdermally into the skin. Here, we used six formulae for elastic liposomes consisting of DOTAP as a cationic lipid and $\mathrm{NaChol}$ or Tween 80 as an edge activator (Table 1): DOTAP liposomes containing Tween 80 at 5, 10, and 15\% (w/w) (T5-, T10-, and T15-liposomes) and DOTAP liposomes containing NaChol at 5, 10, and 15\% (w/w) (C5-, C10-, and C15-liposomes). DOTAP liposomes without an edge activator were used as a control.

Average diameter and $\zeta$-potential of DOTAP liposome were about $110 \mathrm{~nm}$ and $40 \mathrm{mV}$, respectively (Table 1 ). The addition of $\mathrm{NaChol}$ to the formulation of DOTAP liposome decreased the size after preparation of elastic liposome and increased the $\zeta$-potential. C5-, C10-, and C15-liposomes were $80-90 \mathrm{~nm}$ in size and had $\zeta$-potential of $42-52 \mathrm{mV}$. On the other hand, the addition of Tween 80 decreased both the size and the $\zeta$-potential, and T5-, T10-, and T15-liposomes were about $80-90 \mathrm{~nm}$ in size and had $\zeta$-potential of about $26-$ $36 \mathrm{mV}$.

Next, we confirmed the deformability of the elastic liposomes. As shown in Figure 1, the elasticity of liposomes containing Tween 80 peaked at 10\% (w/w) Tween 80 content and was reduced at $15 \%(\mathrm{w} / \mathrm{w})$. T10-liposomes showed 2.7fold-higher elasticity than DOTAP liposomes. In contrast, the elasticity of liposomes containing $\mathrm{NaChol}$ was increased with an increase of $\mathrm{NaChol}$ content, and C15-liposome showed 2.4-fold-higher elasticity than DOTAP liposome. These findings indicate that the optimal amount of edge activator for elasticity of DOTAP liposome differed between the liposomes containing $\mathrm{NaChol}$ and Tween 80.

In the following step, we prepared elastic lipoplex by mixing the liposomes with siRNA. The sizes of DOTAP, C5-, C10-, and C15-lipoplexes were about 1,700-1,860 nm, and those of T5-, T10-, and T15-lipoplexes were about 550$780 \mathrm{~nm}$. Notably, the addition of Tween 80 was observed to reduce the size of the lipoplexes. This might be due to effect of polyoxyethylene on the surface of elastic lipoplex containing Tween 80 .

Next, we investigated the silencing effects of luciferase mRNA and cytotoxicity in SiHa-Luc cells after transfection of the lipoplexes with Luc siRNA (Figures 2(a) and 2(b)). 


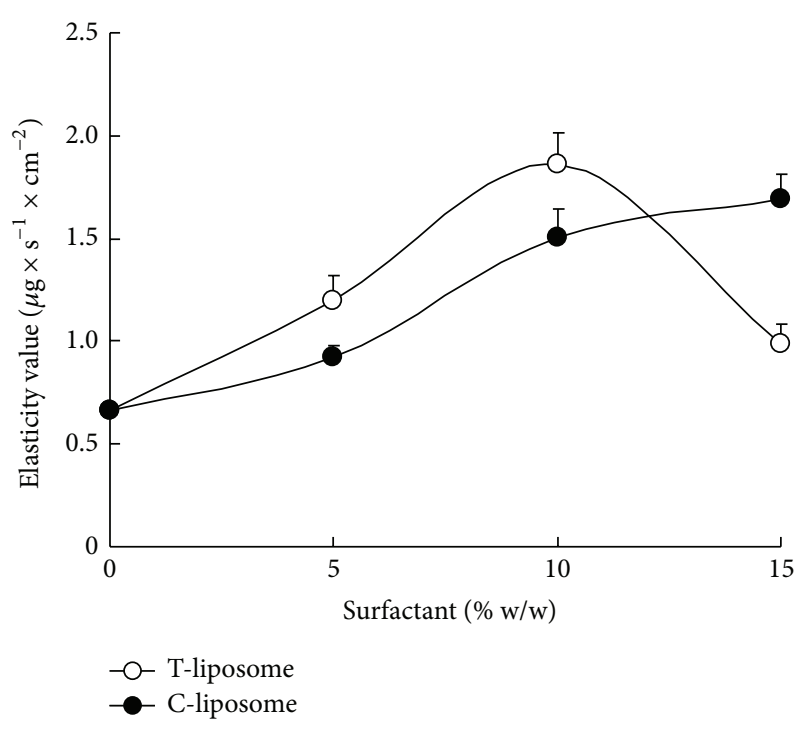

FIgURE 1: Effect of Tween 80 or NaChol content (w/w) on elasticity values of cationic elastic liposomes. Elasticity values were calculated by $J_{\text {flux }} \times\left(r_{v} / r_{p}\right)^{2}$. Each value represents the mean \pm S.D. $(n=3)$.

Although all formulations showed the suppression of luciferase activity without cytotoxicity, an increase of Tween 80 content (T15-liposome) decreased the silencing effect by elastic lipoplex. As a control, lipofectamine RNAiMax, a commercially available transfection reagent, induced strong suppression by siRNA transfection.

To examine the skin permeability of elastic liposomes, we applied rhodamine-labeled elastic liposomes on excised mouse skin for $6 \mathrm{~h}$ and observed their localization by confocal microscopy. Figure 3(a) shows horizontal sections of mouse skin on which the liposomes were applied. The photographs represent sliced images of mouse skin from the surface and up to a thickness of $50 \mu \mathrm{m}$. When rhodamine-labeled DOTAP liposome was applied on the skin, the fluorescence was observed until a thickness of $15 \mu \mathrm{m}$ (Figure 3(a)). However, the liposomes containing $\mathrm{NaChol} \mathrm{improved} \mathrm{skin} \mathrm{penetration}$ with an increase in NaChol content, and fluorescence in the skin after application with C15-liposome was strongly observed up to a depth of $50 \mu \mathrm{m}$. Furthermore, the liposomes containing Tween 80 also showed increased skin penetration compared with DOTAP liposomes, but an increase of Tween 80 content decreased skin penetration. Fluorescence in skin after the application of T5-, T10-, and T15-liposomes was observed at thicknesses of 45,30 , and $35 \mu \mathrm{m}$, respectively. These findings indicate that C15- and T5-liposomes could deeply penetrate into mouse skin.

Finally, we applied the elastic lipoplexes on mouse skin (Figure 3(b)). The photographs represent sliced images of mouse skin from the surface and up to a thickness of $70 \mu \mathrm{m}$. In mouse skin on which naked FAM-labeled siRNA solution was applied, the fluorescence was weakly observed at around $25 \mu \mathrm{m}$ in thickness. In contrast, elastic lipoplexes containing NaChol showed high skin penetration of both siRNA and liposomes with an increase of $\mathrm{NaChol}$ content,

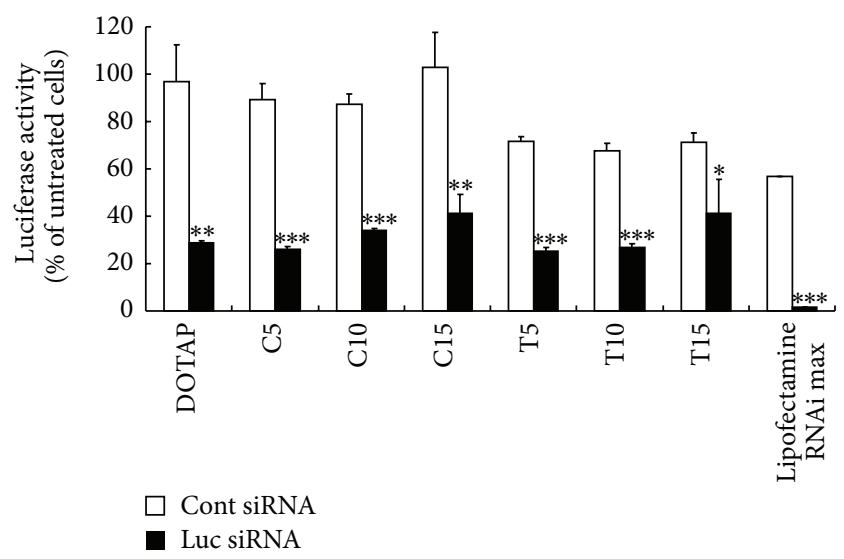

(a)

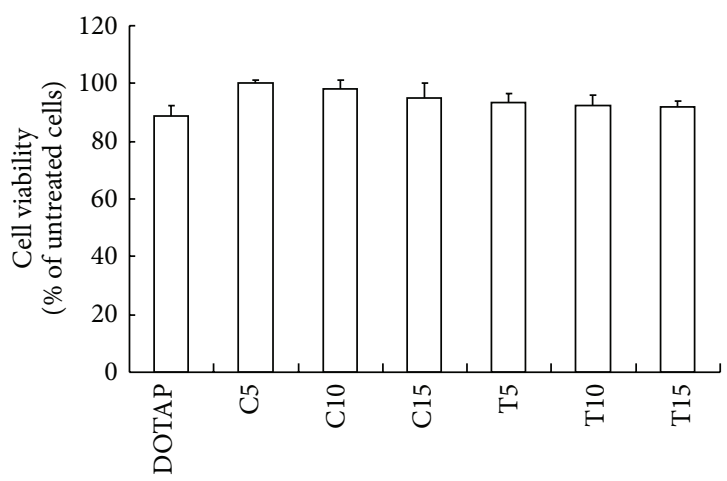

(b)

FIGURE 2: The suppression of luciferase activity (a) and cytotoxicity (b) after siRNA transfection by elastic cationic liposomes. In (a), cationic lipoplexes were added to FL-SiHa cells at $50 \mathrm{nM}$ siRNA. The luciferase assay was carried out $48 \mathrm{~h}$ after incubation of the lipoplexes. Statistical significance was evaluated by Student's $t$-test. ${ }^{*} P<0.05,{ }^{* *} P<0.01$, and ${ }^{* * *} P<0.001$, compared with lipoplex of Cont siRNA. In (b), cytotoxicity was evaluated $48 \mathrm{~h}$ after transfection. In (a) and (b), each column represents the mean \pm S.D. $(n=3)$.

and the fluorescence of FAM-labeled siRNA and rhodaminelabeled liposomes after application with C15-lipoplex was observed at a thickness of around $70 \mu \mathrm{m}$ although the size of C15-lipoplex was large (about $1.8 \mu \mathrm{m}$ ). Moreover, the elastic lipoplex containing Tween 80 also showed improvement of skin penetration. Among the lipoplexes containing Tween 80 , T5-lipoplex $(600 \mathrm{~nm})$ showed improved skin penetration, and the fluorescence of both siRNA and liposomes was observed at around a depth of $70 \mu \mathrm{m}$. The fluorescence of FAM-labeled siRNA after application of C15- and T5lipoplexes largely merged with that of rhodamine-labeled C15- and T5-liposomes, respectively, indicating that siRNA penetrated into the skin as a lipoplex. From these findings, the elastic liposomes might squeeze between cells in the stratum corneum and penetrate the intact skin in vivo through a transcutaneous hydration gradient because the elastic liposome was shown to have high stress-dependent adaptability [16]. In the liposomes containing $\mathrm{NaChol,} \mathrm{C15-liposome} \mathrm{and}$ 


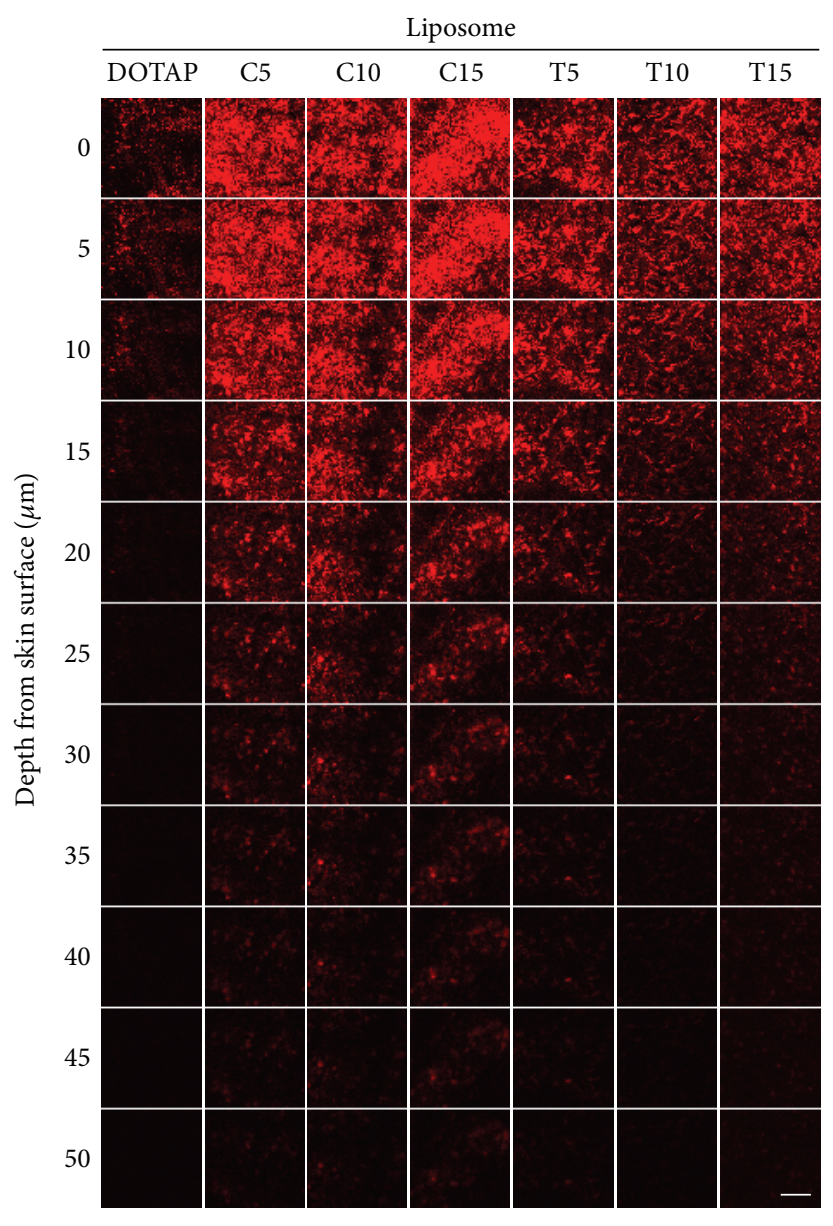

(a)

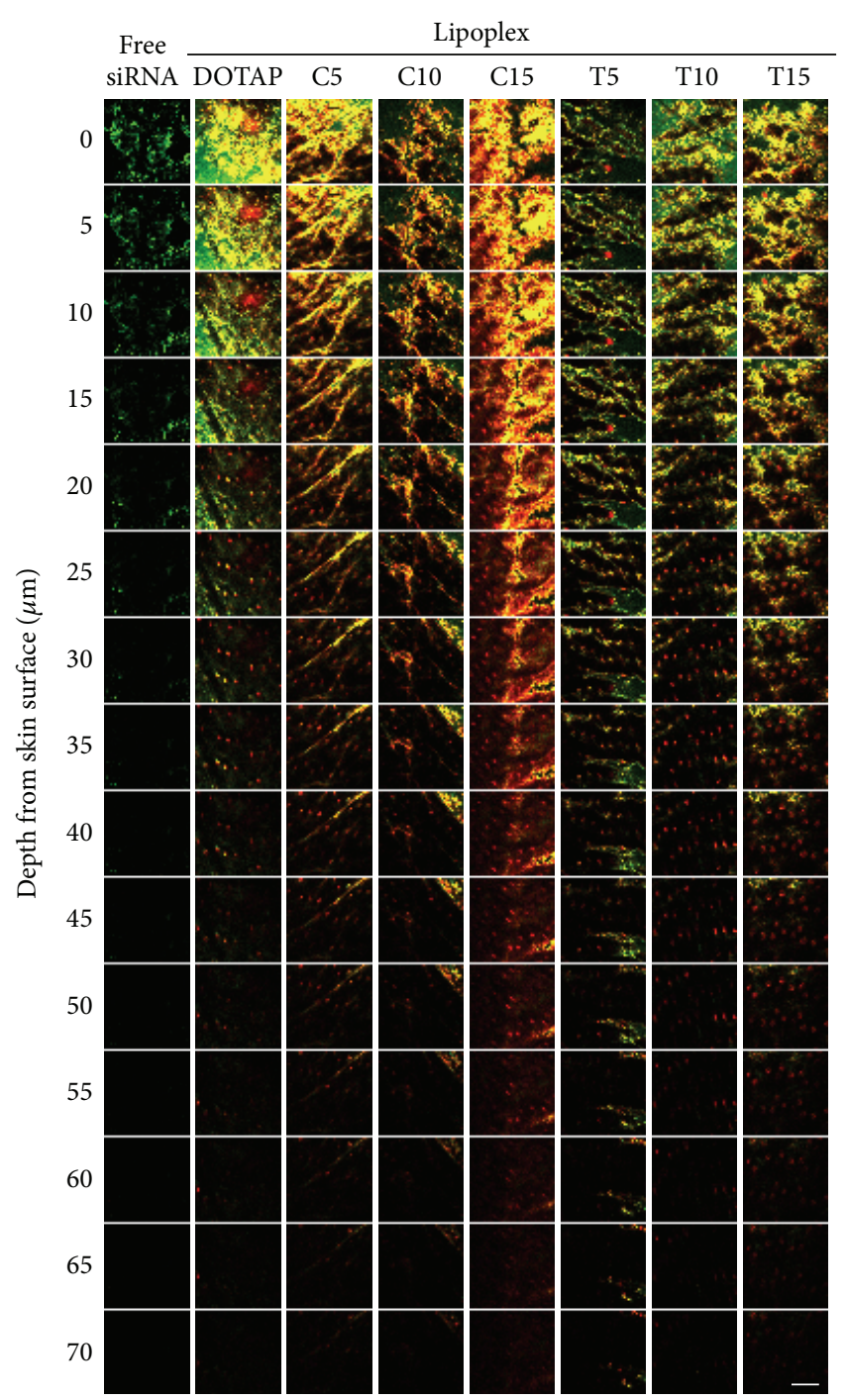

(b)

FIgure 3: Permeability of rhodamine-labeled liposomes (a) and lipoplexes of FAM-labeled siRNA and rhodamine-labeled liposome (b) into mouse skin after topical application for $6 \mathrm{~h}$. In (a), the localizations of rhodamine-labeled liposome are shown as red signals. In (b), the localizations of FAM-labeled siRNA and rhodamine-labeled liposome are shown as green and red signals, respectively. Scale bar $=200 \mu \mathrm{m}$.

lipoplex showed the highest elasticity and skin penetration, respectively; in contrast, in the liposomes containing Tween 80, T10-liposome showed the highest elasticity, but T5lipoplex showed the highest skin penetration, indicating that the optimal amount of Tween 80 for elasticity and penetration might differ between liposome and lipoplex although we did not measure the elasticity of elastic lipoplex.

In pDNA delivery, $\mathrm{NaChol,} \mathrm{NaDChol}$, and Tween 80 have often been used as an edge activator of elastic liposomes. Ultradeformable neutral liposome composed of egg phosphatidylcholine (ePC)/NaChol or ePC/NaDChol displayed the highest level of in vivo transdermal pDNA absorption [8]. Ultradeformable cationic liposomes composed of DOTAP/DOPE/NaDChol [5] and DOTAP/NaChol [6] have been used for the topical delivery of pDNA and antisense oligonucleotide, respectively. However, there is little information about the effect of an edge activator on elastic liposomes for transdermal siRNA delivery in vivo. Geusens et al. reported that ultradeformable cationic liposomes composed of DOTAP and NaChol at a ratio of 6/1 (w/w) (about $14 \%(w / w))$ showed a high gene knock-down effect in melanoma cells by siRNA [9]; however, in in vivo siRNA transfer, they reported that SECosome (surfactant-ethanolcholesterol) consisted of DOTAP, NaChol, and cholesterol at a ratio of $6 / 1 / 1(\mathrm{w} / \mathrm{w} / \mathrm{w})$ in $30 \%$ ethanol, penetrated into epidermis of excised human skin, and suggested that the combination of ethanol and sodium cholate was needed for effective penetration of lipoplexes into skin [17]. In this study, we prepared elastic liposomes containing $\mathrm{NaChol}$ or Tween 80 as an edge activator and found that C15- and T5lipoplexes of siRNA showed high in vitro gene suppression and skin penetration. These findings suggested that $\mathrm{C} 15-$ and T5-liposomes are outstanding tools for transdermal siRNA delivery in mouse skin. 


\section{Conclusion}

In this study, we could develop elastic cationic liposomes for siRNA skin delivery. Our results suggest that $15 \%(\mathrm{w} / \mathrm{w})$ $\mathrm{NaChol}$ or $5 \%(\mathrm{w} / \mathrm{w})$ Tween 80 is the most effective formulation of DOTAP-based liposome for transdermal siRNA delivery. Further study should be performed to examine the effect of gene expression after in vivo siRNA application.

\section{Conflict of Interests}

There is no conflict of interests regarding the publication of this paper.

\section{Acknowledgments}

This project was supported in part by a Grant-in-Aid for Young Scientists (B), Japan Society for the Promotion of Science (KAKENHI Grant no. 23790203), the Advanced Research for Medical Products Mining Programme of the NIBIO, and the Science Research Promotion Fund from the Promotion and Mutual Aid Corporation for Private Schools of Japan.

\section{References}

[1] P. Resnier, T. Montier, V. Mathieu, J. P. Benoit, and C. Passirani, "A review of the current status of siRNA nanomedicines in the treatment of cancer," Biomaterials, vol. 34, pp. 6429-6443, 2013.

[2] P. Kubowicz, D. Zelaszczyk, and E. Pekala, "RNAi in clinical studies," Current Medicinal Chemistry, vol. 20, pp. 1801-1816, 2013.

[3] B. Geusens, T. Strobbe, S. Bracke et al., "Lipid-mediated gene delivery to the skin," European Journal of Pharmaceutical Sciences, vol. 43, no. 4, pp. 199-211, 2011.

[4] M. B. R. Pierre and I. Dos Santos Miranda Costa, "Liposomal systems as drug delivery vehicles for dermal and transdermal applications," Archives of Dermatological Research, vol. 303, no. 9, pp. 607-621, 2011.

[5] J. Li, X. Li, Y. Zhang et al., "Gene therapy for psoriasis in the K14VEGF transgenic mouse model by topical transdermal delivery of interleukin-4 using ultradeformable cationic liposome," Journal of Gene Medicine, vol. 12, no. 6, pp. 481-490, 2010.

[6] S. T. Kim, K.-M. Lee, H.-J. Park, S.-E. Jin, W. S. Ahn, and C.-K. Kim, "Topical delivery of interleukin-13 antisense oligonucleotides with cationic elastic liposome for the treatment of atopic dermatitis," Journal of Gene Medicine, vol. 11, no. 1, pp. 26-37, 2009.

[7] M. M. A. Elsayed, O. Y. Abdallah, V. F. Naggar, and N. M. Khalafallah, "Lipid vesicles for skin delivery of drugs: reviewing three decades of research," International Journal of Pharmaceutics, vol. 332, no. 1-2, pp. 1-16, 2007.

[8] E. H. Lee, A. Kim, Y.-K. Oh, and C.-K. Kim, "Effect of edge activators on the formation and transfection efficiency of ultradeformable liposomes," Biomaterials, vol. 26, no. 2, pp. 205-210, 2005.

[9] B. Geusens, J. Lambert, S. C. De Smedt, K. Buyens, N. N. Sanders, and M. Van Gele, "Ultradeformable cationic liposomes for delivery of small interfering RNA (siRNA) into human primary melanocytes," Journal of Controlled Release, vol. 133, no. 3, pp. 214-220, 2009.
[10] Z. Ma, J. Li, F. He, A. Wilson, B. Pitt, and S. Li, "Cationic lipids enhance siRNA-mediated interferon response in mice," Biochemical and Biophysical Research Communications, vol. 330, no. 3, pp. 755-759, 2005.

[11] M. Sioud and D. R. Sørensen, "Cationic liposome-mediated delivery of siRNAs in adult mice," Biochemical and Biophysical Research Communications, vol. 312, no. 4, pp. 1220-1225, 2003.

[12] D. R. Sørensen, M. Leirdal, and M. Sioud, "Gene silencing by systemic delivery of synthetic siRNAs in adult mice," Journal of Molecular Biology, vol. 327, no. 4, pp. 761-766, 2003.

[13] Y. Hiruta, Y. Hattori, K. Kawano, Y. Obata, and Y. Maitani, "Novel ultra-deformable vesicles entrapped with bleomycin and enhanced to penetrate rat skin," Journal of Controlled Release, vol. 113, no. 2, pp. 146-154, 2006.

[14] K. G. Lau, Y. Hattori, S. Chopra et al., "Ultra-deformable liposomes containing bleomycin: in vitro stability and toxicity on human cutaneous keratinocyte cell lines," International Journal of Pharmaceutics, vol. 300, no. 1-2, pp. 4-12, 2005.

[15] Y. Hattori, T. Nakamura, H. Ohno, N. Fujii, and Y. Maitani, "siRNA delivery into tumor cells by lipid-based nanoparticles composed of hydroxyethylated cholesteryl triamine," International Journal of Pharmaceutics, vol. 443, pp. 221-229, 2013.

[16] G. Cevc, A. Schätzlein, and H. Richardsen, "Ultradeformable lipid vesicles can penetrate the skin and other semi-permeable barriers unfragmented. Evidence from double label CLSM experiments and direct size measurements," Biochimica et Biophysica Acta, vol. 1564, no. 1, pp. 21-30, 2002.

[17] B. Geusens, M. Van Gele, S. Braat et al., "Flexible nanosomes (SECosomes) enable efficient siRNA delivery in cultured primary skin cells and in the viable epidermis of ex vivo human skin," Advanced Functional Materials, vol. 20, no. 23, pp. 40774090, 2010. 

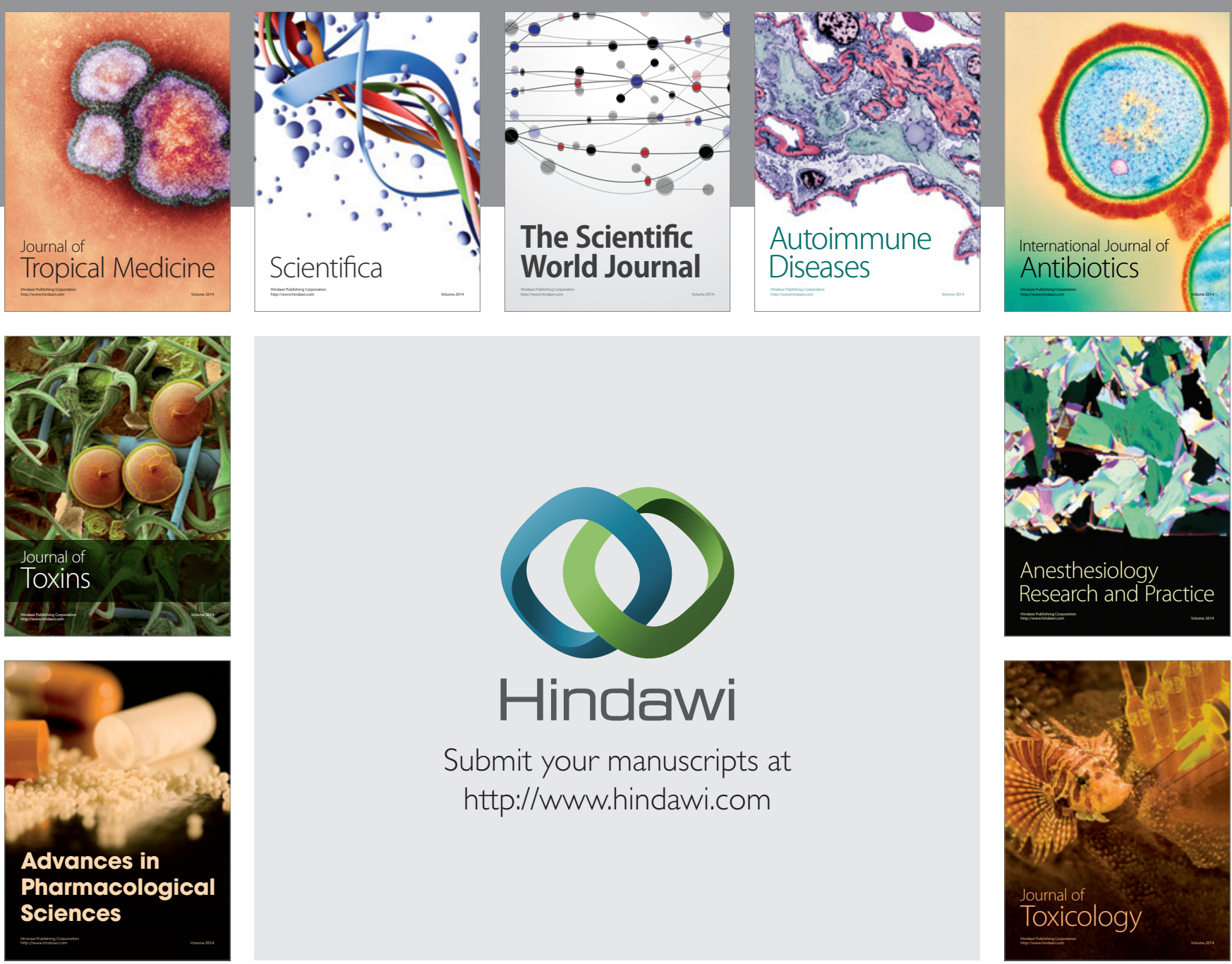

\section{Hindawi}

Submit your manuscripts at

http://www.hindawi.com
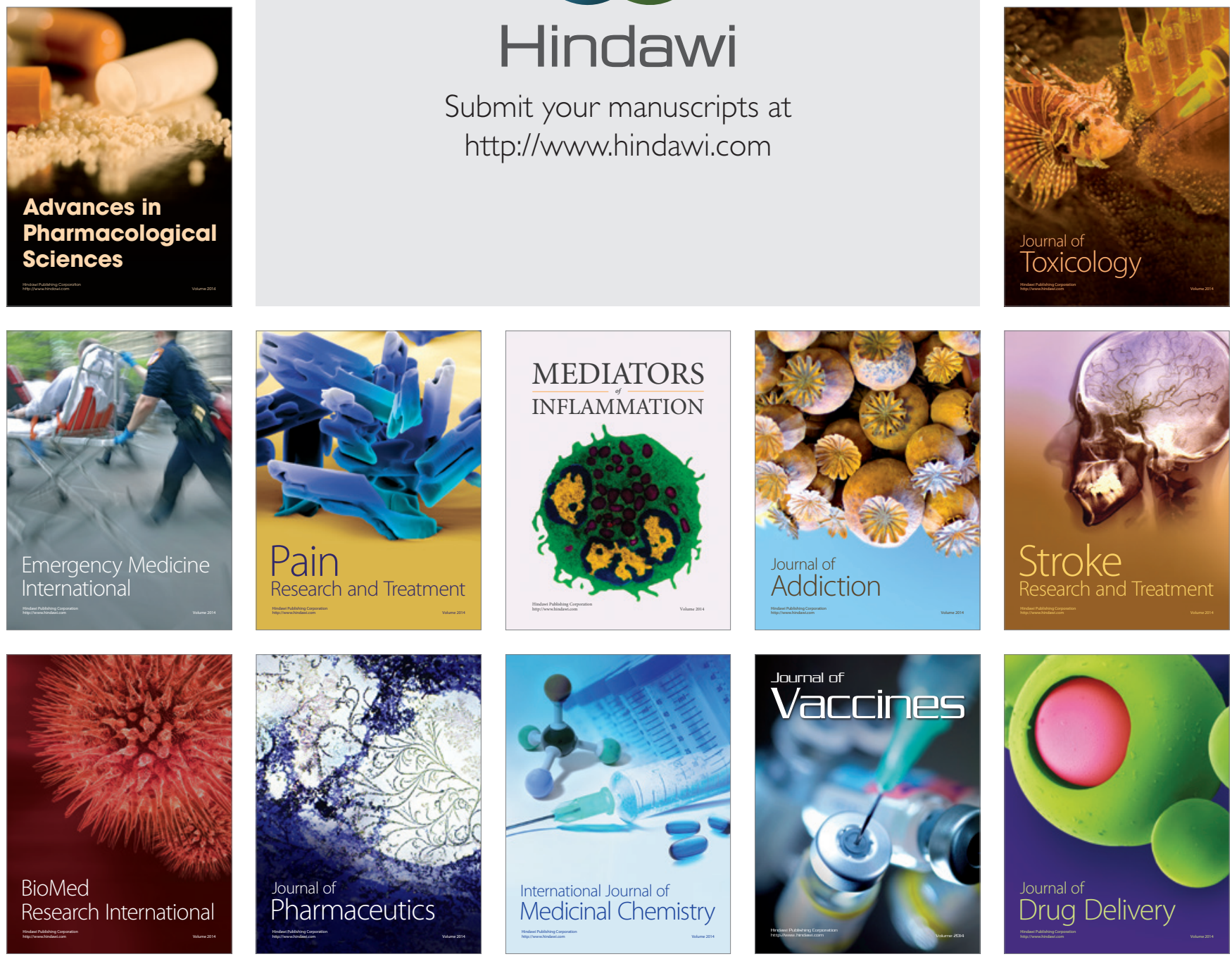